\title{
Metabolites from intestinal microbes shape Treg
}

\author{
Cell Research (2013) 23:1339-1340. doi:10.1038/cr.2013.125; published online 10 September 2013
}

\begin{abstract}
Intestinal bacterial metabolites are an important communication tool between the host immune system and the commensal microbiota to establish mutualism. In a recent paper published in Science, Wendy Garrett and her colleagues report an exciting role of the three most abundant microbial-derived shortchain fatty acids (SCFA), acetic acid, propionic acid and butyric acid, in colonic regulatory $\mathbf{T}$ cell (cTreg) homeostasis.
\end{abstract}

A number of studies have shown that increased cTreg numbers and their immunoregulatory function are promoted by the presence of commensal intestinal microbes (either individual species such as Bacteroides fragilis [1], defined benign consortia of bacteria such as the altered Schaedler flora [2] or groups of Clostridia [3]). In a recent paper in Science, Garrett and colleagues report how these effects are generated through molecular exchanges between the host and the enormous load of microbes carried in the lower intestine [4].

Smith et al. [4] investigated the role of SCFA, which are bacterial fermentation products produced by a wide variety of bacteria through anaerobic acidogenic pathways. SCFA released by colonic bacteria have long been known to be important as a carbon source for colonic epithelial cells [5]. From this new work we can now see that signaling effects of SCFA also regulate cTreg homeostasis.

Microbiota-derived SCFA were found to increase total (thymic-derived) cTreg numbers. The homing characteristics to the colon and the reg- ulatory functions of these cells (such as IL-10 production) were also enhanced through SCFA treatment.

These effects are mediated by the G-protein-coupled free fatty acid receptor 43 (GPR43). Using mice that are genetically deficient in this receptor, Smith et al. showed that this signaling pathway is responsible for the increased cTreg numbers in vivo and that signaling by SCFA reduces the susceptibility to chronic intestinal inflammation. As they found GPR43 expression on cTreg (compared with lower GPR43 expression on Treg from other sites) this may be a direct effect, e.g. alterations in histone deacetylation. However, other cell types in the GI tract also express GPR43, including enteroendocrine cells and other leukocytes, therefore indirect effects are not yet excluded. In fact, Atarashi and colleagues have recently published their studies of how Clostridial species induce cTreg [6]. They found that bacterial-derived SCFA stimulate epithelial cells to produce TGF $\beta$, contributing to Treg differentiation and expansion.

Whereas other species-specific bacterial molecules, such as B. fragilisderived PSA, have previously been demonstrated to have immunomodulatory functions [2], the report by Smith et al. is an elegant demonstration of the ubiquitous and pervasive bacterial metabolites that impact on the mucosal immune system. There is really a rather promiscuous exchange of metabolites between the microbiota and the host, with metabolic pathways that require components of both eukaryotic and prokaryotic cells. Bile acids are a great example of such a mixed pathway, where a dysbiosis caused by obesity promotes liver cancer through alterations in the microbial bile acid metabolism [7]. Although Smith et al. do not see any SCFA-mediated effects on central Treg compartments (outside the colon), other bacterial metabolites that reach systemic sites likely modulate adaptive or innate immune cell function at systemic sites. This may eventually rationalize the observed increased incidence of intestinal inflammation and systemic immune-mediated disorders such as autoimmune or allergic diseases (Figure 1), which are often linked to changes within the microbiota due to diet or antibiotic use [8].

A clinical situation in which the colon faces a deficiency of SCFA happens after surgery that diverts the fecal stream into a stoma bag, leaving the distal colon without its normal contents. This operation may be carried out to protect a low surgical anastomosis after removal of a tumor. The result is that the defunctioned colon frequently becomes inflamed, a condition recognized as 'diversion colitis'. In some cases, treatment with SCFA has been able to treat the condition successfully [9]. The lack of SCFA as a carbon source for colonocytes was previously considered as a key factor in the aetiopathogenesis of the condition, although this will need to be reviewed in the light of the new data on the effects of SFCA on colonic Treg numbers and function.

Our colonic health depends on our intestinal microbiota and what we feed them. Changes in Western dietary patterns, e.g., due to reduced intake of 


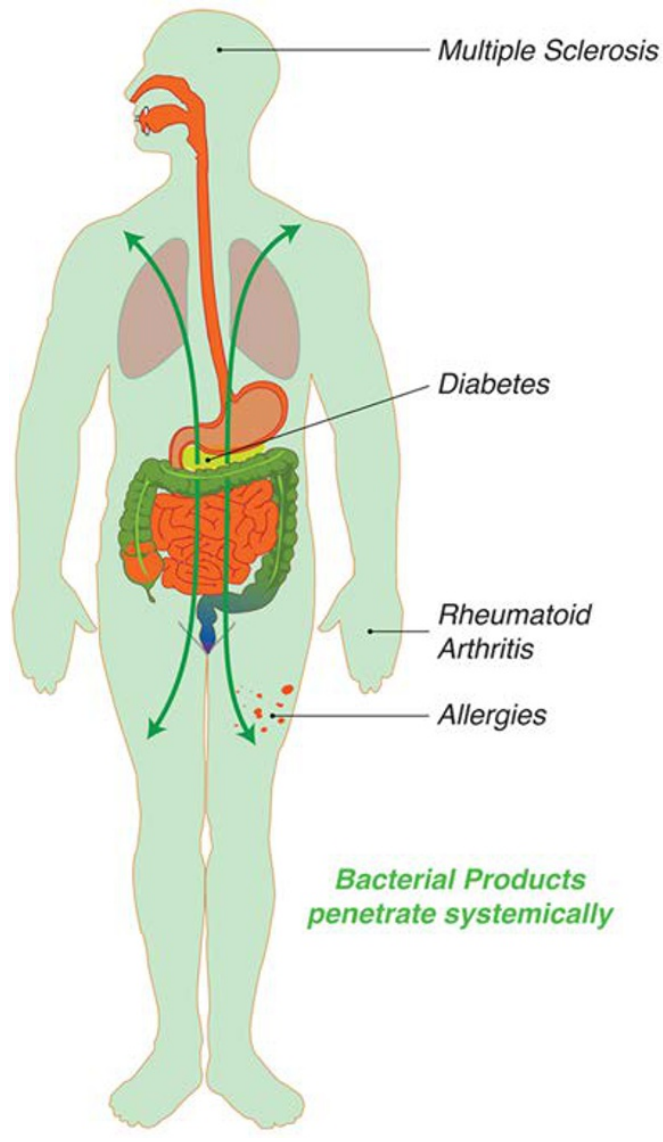

Figure 1 Bacterial metabolites that reach systemic sites likely modulate adaptive or innate immune cell function at systemic sites. This may eventually rationalize the observed correlation of microbiota composition and susceptibility to systemic immunemediated disorders such as autoimmune or allergic diseases.

plant fibers, might drastically impact the production of SCFA within the intestine. Furthermore, Smith et al. demonstrate a direct effect of antibiotic (vancomycin) treatment on SCFA levels, which in turn affects intestinal immune regulation by reducing the number of cTreg. derived to challenge the notion that the existence of higher organisms was irrevocably linked to their associated microbiotas [10]. Although the germ-free program succeeded [11], it has provided us with powerful tools to show that the original notion was justified: pervasive metabolic interactions and signaling make us the sum of our prokaryotic and eukaryotic cellular components.

Markus B Geuking ${ }^{1}$, Kathy D McCoy ${ }^{1}$, Andrew J Macpherson ${ }^{1}$

${ }^{1}$ University of Bern, Maurice Müller Laboratories $(D K F)$, University Clinic for Visceral Surgery and Medicine, Murtenstrasse 35, CH-3010 Bern, Switzerland

Correspondence: Andrew J Macpherson

E-mail: andrew.macpherson@insel.ch Tel: +41316328025

\section{References}

1 Round JL, Mazmanian SK. Proc Natl Acad Sci USA 2010; 107:12204-12209.

2 Geuking MB, Cahenzli J, Lawson MA, et al. Immunity 2011; 34:794-806.

3 Atarashi K, Tanoue T, Shima T, et al. Science 2011; 331:337-341.

4 Smith PM, Howitt MR, Panikov N, et al. Science 2013; 341:569-573.

5 Roediger WE. Gastroenterology 1982; 83:424-429.

6 Atarashi K, Tanoue T, Oshima K, et al. $\mathrm{Na}$ ture 2013; 500:232-236.

7 Yoshimoto S, Loo TM, Atarashi K, et al. Nature 2013; 499:97-101.

8 Markle JG, Frank DN, Mortin-Toth S, et al. Science 2013; 339:1084-1088.

9 Kiely EM, Ajayi NA, Wheeler RA, et al. $J$ Pediatr Surg 2001; 36:1514-1517.

10 Pasteur L. Comp Rend 1885; 100:69.

11 Glimstedt G. Acta Pathol Microbiol Scand Suppl 1936; 30:1-295. 